Trans

continentales
Transcontinentales

Sociétés, idéologies, système mondial

$8 / 9 \mid 2010$

Des migrations aux circulations transnationales

\title{
Les migrants de travail ouzbeks à Moscou
}

Une aventure risquée

Sophie Massot

\section{CpenEdition}

Journals

Édition électronique

URL : http://journals.openedition.org/transcontinentales/780

DOI : $10.4000 /$ transcontinentales. 780

ISBN : 978-2-7351-1557-0

ISSN : $1775-397 X$

Éditeur

Editions de la maison des sciences de l'homme

Édition imprimée

Date de publication : 31 décembre 2010

ISSN : 1950-1684

Référence électronique

Sophie Massot, «Les migrants de travail ouzbeks à Moscou », Transcontinentales [En ligne], 8/9 | 2010, document 16, mis en ligne le 31 décembre 2010, consulté le 08 septembre 2020. URL : http:// journals.openedition.org/transcontinentales/780; DOI : https://doi.org/10.4000/transcontinentales. 780

Ce document a été généré automatiquement le 8 septembre 2020

Tous droits réservés 


\section{Les migrants de travail ouzbeks à Moscou}

Une aventure risquée

Sophie Massot

1 «Partir à l'étranger pour mieux vivre en Ouzbékistan»: cette formule paradoxale résume l'enjeu de l'émigration en provenance d'Ouzbékistan'1 . La plupart des migrants quittent temporairement leur famille pour se rendre à l'étranger afin de profiter de salaires bien plus avantageux que dans leur pays d'origine, envisageant d'y revenir une fois leurs économies réalisées.

2 L'analyse de ce phénomène migratoire, massif puisqu'il pourrait concerner jusqu'à $30 \%$ de la population ouzbékistanaise ${ }^{2}$, se fera à partir du point de vue des migrants euxmêmes. Cette migration du mieux-vivre, qui n'est pas une mobilité de survie, concerne essentiellement des hommes de milieu socio-économique moyen. À travers leurs histoires de vie, leurs expériences et leurs jugements, sera abordée l'immigration vers la Russie, et plus particulièrement vers Moscou. La recherche porte spécifiquement sur les migrants ouzbeks, et non sur les multiples groupes ethniques présents en Ouzbékistan. Les États concernés ont une attitude ambiguë à l'égard de ce phénomène relativement nouveau. Face à cette hémorragie démographique, le régime d'Islam Karimov minimise l'ampleur de l'émigration et fustige les exilés, tout en profitant des retombées économiques de ces mouvements de population. De son côté, la Russie ne ferme pas ses frontières mais ne décourage pas la xénophobie grandissante de la population à l'encontre des Centrasiatiques. Alors comment expliquer l'engouement des migrants ouzbeks pour cette destination?

\section{La Russie, ou l'importance de « l'étranger proche »}

Les rapports entre l'Ouzbékistan et la Russie sont anciens et commencent bien avant l'époque soviétique. Dans le cadre d'une politique tsariste expansionniste, l'actuel Ouzbékistan fut annexé par les troupes russes, dès la seconde moitié du XIX ${ }^{e}$ siècle. En 
1924, les Soviétiques créent la République d'Ouzbékistan. Depuis la chute de l'URSs, la Russie en reste un partenaire primordial.

Au niveau politique, le partenariat est décisif, comme en témoignent les nombreuses rencontres entre les représentants des deux pays et les multiples accords bilatéraux. Pour ne citer que quelques exemples, notons qu'un accord militaire, conséquence directe des événements d'Andijan³, a été signé le 14 novembre 2005 entre Vladimir Poutine et Islam Karimov, qui s'assurent ainsi réciproquement assistance mutuelle en cas d'agression ${ }^{4}$. Par ailleurs, l'Ouzbékistan a rejoint en 2005-2006 l'Organisation du traité de sécurité collective (CSTO) ${ }^{5}$ et la Communauté économique eurasienne

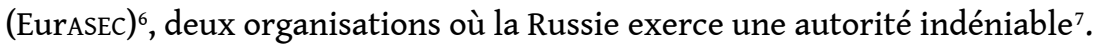

5 Au niveau économique, la Russie est le premier partenaire de l'Ouzbékistan, tant pour les exportations que pour les importations ${ }^{8}$; en 2007 , les pays de la CEI ont contribué pour un tiers au commerce extérieur de l'Ouzbékistan'. En décembre 2005, un accord sous la forme d'un trade-house a été conclu afin de développer le partenariat commercial et économique entre les deux États ${ }^{10}$.

6 Au-delà de ces relations formelles, tout un ensemble de liens se sont tissés entre les deux sociétés. Les Russes constituaient à la fin de l'époque soviétique la minorité la plus importante $(8,3 \%)$ du pays. À l'indépendance, nombre d'entre eux ont gagné la Russie, mais ils représenteraient encore environ $5,7 \%$ de la population ${ }^{11}$.

7 La langue russe garde le statut de lingua franca pour l'ensemble des républiques d'URSS. Si l'ouzbek a été promu langue officielle d'État avant même que l'indépendance ne soit proclamée, il n'est compris que par $65,6 \%$ de la population ${ }^{12}$ tandis que plus d'une cinquantaine de langues vernaculaires sont parlées en Ouzbékistan ${ }^{13}$. Septembre 2005 signe le passage définitif des caractères cyrilliques à l'alphabet latin et à l'emploi de la langue ouzbèke dans les institutions étatiques et administratives ${ }^{14}$. Néanmoins, le russe reste aujourd'hui la langue véhiculaire en Ouzbékistan, bien qu'aucun statut officiel ne lui soit accordé ${ }^{15}$. Sur le plan culturel, les domaines artistiques et de divertissement sont particulièrement marqués par l'influence russe. Les chaînes de télévision russes ${ }^{16}$, surtout $\mathrm{NTV}^{17}$, sont très regardées et constituent pour beaucoup d'habitants du pays une source d'information jugée plus fiable que celle offerte par les chaînes nationales.

8 L'hégémonie russe est grande dans tous les domaines, et le rapport entre les deux pays n'est pas placé sous le signe de l'égalité, l'ancien « frère russe » restant davantage une référence qu'un simple partenaire. L'héritage soviétique est donc fort, la Russie continuant de manière générale à entretenir des relations privilégiées avec les pays issus de l'ex-URSS, réunis sous le terme bližnee zarubež’e [ $r^{18}{ }^{18}$ étranger proche, dont sont exclus les Pays Baltes] et s'opposant au dal'nee zarubeže [r. : étranger lointain]. De la même manière, les migrants n'estiment a priori pas leur départ vers la Russie comme un déracinement. Moscou n'est ainsi pas véritablement envisagée par mes interlocuteurs en Ouzbékistan comme une ville totalement étrangère. Norbek ${ }^{19}$, originaire de Samarkand, qui connaît bien la Russie pour y faire régulièrement $\mathrm{du}$ commerce, explique :

Mon beau-frère habite Cincinnati, mais il s'est endetté pour aller là-bas. Et, il ne peut pas revenir ici tant qu'il n'a pas gagné assez d'argent, sinon, ça ne sert à rien. Je ne suis jamais allé aux Usa, et je n'envisage pas d'y aller, même si j'ai des amis làbas. J'y avais pensé pour les études de mon fils, mais il est jeune encore [le garçon concerné a douze ans]. Il parle anglais donc ce serait bien, mais sa mère a peur pour lui [rires]. Il est très bon en football et j'aimerais qu'il fasse une carrière sportive, ce 
serait très intéressant. Je vais donc lui faire passer les examens d'entrée pour un collège à Moscou. Il parle russe sans difficulté, donc ce ne sera pas un problème pour lui. Ce n'est pas loin. C'est l'ex-Urss, quoi !

9 Effectivement, l'ancien bloc soviétique est considéré par de nombreux Ouzbékistanais comme constituant une unité de valeurs. Quitter l'Ouzbékistan pour la Fédération de Russie n'est pas vécu comme un exil. Le fils de Norbek n'est pas censé être perdu dans cet univers moscovite, considéré, malgré le nombre de kilomètres qui les sépare, comme proche de Samarkand. Ce qui inquiète sa mère dans ce projet d'étude est uniquement la séparation de la sphère familiale et non pas l'environnement socioculturel.

10 La capitale russe, comme d'autres villes du territoire, est pourtant le théâtre de nombreuses agressions touchant notamment les migrants centrasiatiques. L'ancien projet communiste des peuples frères a été largement oublié, au profit d'une xénophobie omniprésente, qui se traduit parfois par des actes violents envers les nonRusses. Toutefois, en dépit de la peur qui s'empare de la plupart des migrants à l'idée de sortir dans le métro ou dans les rues moscovites, la Russie garde, envers et contre tout, son image de destination « facile».

11 Cette "proximité» est davantage symbolique que géographique, puisque 3000 kilomètres environ séparent Tachkent de Moscou ${ }^{20}$. Les moyens de transport pour se rendre dans la capitale russe sont divers ${ }^{21}$ et jugés peu onéreux ${ }^{22}$, ce qui favorise les départs et rend possible les séjours saisonniers.

\section{Déroulement de la recherche de terrain}

12 Une étude de terrain est toujours une entreprise délicate à mener. Le chercheur doit constamment adapter ses méthodes, car on observe ou on n'interroge pas de la même manière un migrant de retour dans sa famille, tranquillement installé devant un bol de thé, qu'un migrant craignant un contrôle d'identité dans un café de Moscou.

13 La source principale d'information est en effet la parole des migrants, recueillie à travers des entretiens compréhensifs menés en langue russe et complétée par l'observation participante. Les entretiens que je cite ont eu lieu au cours des enquêtes de terrain suivantes : terrain préparatoire en Ouzbékistan en juillet-août 2005, puis à Moscou en avril-mai 2006 et à nouveau en Ouzbékistan en avril-mai 2007²3. Au cours de ces recherches, je me suis donc retrouvée migrante parmi les migrants, bien que pour des motifs bien différents des Ouzbeks et parfois considérés comme fallacieux. Certains imaginaient que mon sujet de recherche n'était qu'un prétexte, le véritable objet de ma venue ne pouvant être que le travail et l'appât du gain, comme eux. Ils me conseillaient alors des moyens pour obtenir un emploi, et comprenaient mal ma réticence. D'autres craignaient que je ne sois en réalité un espion, voire un agent double, Française travaillant pour l'Ouzbékistan et/ou le pays d'accueil.

14 C'est pourquoi persuader les migrants d'accepter un entretien s'avère périlleux et demande beaucoup de persuasion de la part du chercheur, surtout dans les premiers moments du terrain, lorsqu'il ne connaît encore personne et ne peut donc pas se réclamer d'un réseau de relations, et lorsqu'il ne maîtrise pas les codes spécifiques aux migrants du pays d'accueil. L'apprentissage progressif de ces codes permet de mieux 
cerner le monde de référence des migrants et d'approfondir toujours plus les entretiens.

La principale difficulté a été de trouver mes premiers interlocuteurs. Il me faut confier ici certaines idées fausses que j'avais et que le terrain a démenties. Les prénotions et préjugés sont inéluctables, tant la culture d'origine d'un chercheur lui pose des œillères et des a priori dont il n'a pas conscience. Les utiliser comme hypothèses de travail, afin de les voir ou non confirmés par le terrain, permet de s'en affranchir. Deux principales pistes se sont ainsi révélées erronées.

Tout d'abord, je pensais que mon premier terrain de thèse en Ouzbékistan me permettrait non seulement de rencontrer des familles de migrants, mais également que celles-ci pourraient me confier les coordonnées de leur parent parti pour l'étranger. Or si ce travail préalable s'est avéré essentiel pour définir le cadre du sujet et comprendre à la fois les motivations au départ et leurs impacts sur le pays d'accueil, il ne m'a pas permis d'obtenir de contacts avec des migrants partis pour la Russie. Je pensais initialement qu'il s'agissait d'une réticence de la part de certains à me confier le moyen de communiquer avec leur parent exilé. Cela représentait un moyen de contacter des migrants peut-être en situation d'illégalité, dont la famille ne sait pas s'ils sont " présentables », s'ils vivent dans un logement décent, etc. En réalité, il apparait que les familles ne disposent pas de ces coordonnées: les migrants les appellent mais pas l'inverse. Cela répond tout d'abord à une logique économique, les appels depuis l'Ouzbékistan étant plus onéreux que ceux passés depuis la Russie. Par ailleurs, les migrants ne disposent pas toujours d'un téléphone, et sont amenés à changer d'adresse régulièrement. J'ai donc dû renoncer à cette possibilité d'entrer dans les communautés ouzbeks à l'étranger.

Un deuxième préjugé important a été mis à mal. J'estimais, en effet, au regard de l'importance des réseaux sociaux en Ouzbékistan, que les migrants ne manqueraient pas de constituer des réseaux d'entraide. En réalité, il est apparu que ceux-ci ne fonctionnaient pas comme je l'avais envisagé. Il m'a donc fallu lier contact avec des interlocuteurs directement à Moscou et rechercher des lieux privilégiés pour espérer rencontrer des migrants venus d'Ouzbékistan.

\section{Où rencontrer des migrants dispersés, craintifs et casaniers?}

18 Je ne possédais en arrivant dans la capitale russe qu'un seul numéro de téléphone, que m'avait confié un Ouzbek à Samarkand. Il s'agissait de celui d'un de ses frères. La rencontre avec ces migrants (trois frères et leur neveu) fut particulièrement riche. Néanmoins, contrairement à mes attentes, cette famille ne m'a pas présentée ou mise en relation avec d'autres migrants. La fratrie semblait vivre en vase clos, ne sortant du microcosme professionnel que pour rentrer dans son logement. Je n'ai donc pas pu m'appuyer sur un réseau de connaissances pour mener mes premiers entretiens.

19 La fréquentation de certains lieux m'a permis de rencontrer des travailleurs centrasiatiques, à commencer par la gare de Kazan ${ }^{24}$ où arrivent les trains en provenance d'Asie centrale. En réalité, je n'y ai mené qu'un seul entretien et de manière assez fortuite, puisque c'est un Ouzbek qui, après m'avoir aperçue arpenter plusieurs fois les quais, m'a proposé de me vendre une fausse registraciâa ${ }^{25}$, avec « un 
tampon exactement comme ceux de la police ". J'ai, à mon grand étonnement, pu mener plusieurs heures durant un entretien libre avec lui dans un des cafés de la gare, après qu'il eut informé un de ses amis de sa destination, sans doute par crainte de ce qui pouvait lui arriver. Alors qu'il exerçait une activité de faussaire totalement hors-laloi, mais sûrement avec l'accord tacite voire monnayé de la police, il acceptait de me parler de son métier et de sa vie. Cependant, il est le seul avec qui j'ai parlé dans la gare de Kazan, car il me paraissait malaisé d'assaillir de questions les migrants tout juste sortis du train.

Dans un premier temps, je me suis contentée d'observer leur arrivée. J'ai pu lire l'étonnement mêlé de crainte sur les visages des migrants qui ne connaissaient pas Moscou et qui semblaient désorientés et sans repère. Certains de leurs compagnons de route, plus chanceux, étaient attendus par une connaissance et se laissaient donc guider. Ensuite, j'ai pu constater que certains attendaient en groupe discipliné des instructions données par une femme, qui prenait leurs passeports avant de les emmener à l'arrière de la gare, où ils étaient répartis par trois ou quatre dans des taxis. Cela montre qu'une partie de l'aventure migratoire est organisée et structurée par des institutions informelles.

21 J'ai également privilégié les lieux de travail des migrants. Les plus évidemment identifiables étaient les restaurants et cafés ouzbeks, puisque nombre d'entre eux sont répertoriés dans des annuaires et que des recensions sont disponibles sur Internet. J'ai constaté dans plusieurs d'entre eux ${ }^{26}$, non sans surprise, leur haut standing, à la décoration orientalisante et luxueuse, avec une clientèle suffisamment fortunée pour consommer des mets relativement chers. La culture ouzbèke y apparaît sous forme de clichés exotiques: danseuses du ventre aux tenues minimalistes, combats de coq, fontaines recouvertes de mosaïques bleues, serveurs affublés de déguisements soyeux ou encore ceinturés de larges bandeaux de tissus chamarrés. Cette mise en scène reflète mal la réalité ouzbèke. Si les clients sont en majorité des Russes, les serveurs et cuisiniers sont bien souvent des Centrasiatiques. J'ai ainsi pu prendre contact avec certains membres du personnel, me contentant de leur demander de me consacrer du temps pour discuter. Cette situation n'était pas idéale, car mes interlocuteurs étaient inquiets d'évoquer leur situation avec une étrangère sur leur lieu de travail. Certains arguaient de cet obstacle pour éviter tout contact, au moins lors de ma première visite.

Par ailleurs, je me suis rendue dans les différents marchés de la capitale, autres hauts lieux de main-d'œuvre immigrée ${ }^{27}$. De nombreux migrants y sont employés, principalement dans la vente des fruits et légumes et la manutention ${ }^{28}$. Il y a également des petites cantines centrasiatiques et des étals vendant principalement du plov ${ }^{29}$, où les travailleurs ouzbeks viennent parfois se restaurer. Là encore, les premiers contacts n'ont pas été faciles, tant les Centrasiatiques travaillant là craignent d'attirer l'attention. Néanmoins, en revenant plusieurs fois aux mêmes endroits en y consommant des plats ouzbeks, la tension s'est peu à peu apaisée et j'ai pu recueillir d'intéressants témoignages.

23 À une autre occasion, un chercheur russe, connu dans le milieu universitaire pour ses recherches sur les migrations en Russie, m'a invitée à mener avec lui quelques entretiens au marché de Teplyj stan ${ }^{30}$. Il estimait que je n'avais aucune chance de rencontrer par moi-même des Ouzbeks à Moscou et encore moins de parvenir à en tirer quelques bribes d'informations. J'ai ravalé ma fierté et accepté sa proposition. S'étaient jointes à nous une étudiante désireuse de parler français et sa mère, qui craignait que 
sa fille, âgée de 26 ans, ne se rende seule dans des lieux mal famés. Le chercheur, d'ordinaire en costume de ville, m'expliqua qu'il avait ce jour-là endossé un blue-jean pour " passer plus inaperçu », tout en gardant un très chic blazer bleu marine. La leçon de terrain fut désastreuse. À notre arrivée sur le marché, dès qu'il apercevait un commerçant possiblement originaire d'Asie centrale, le chercheur l'assaillait de questions, aussi directives que gênantes - «Ils sont racistes, les Russes, non ? Vous pouvez le dire, on est entre nous », ou encore «Vous gagnez combien? » ou bien « Vous n'êtes pas enregistrés ${ }^{31}$, n'est-ce pas?» - tout en m'intimant de filmer ses échanges avec mon appareil photo.

Les vendeurs interrogés étaient visiblement apeurés mais, craignant de s'attirer des ennuis et ne sachant qui était véritablement ce prétendu chercheur, répondaient malgré tout, usant d'une langue de bois aux mille détours. Les migrants s'attroupaient autour de l'interviewé, intrigués par ce manège hors du commun. Rapidement, c'est un agent de police qui est intervenu pour faire cesser tout cela, expliquant que cela troublait l'ordre du marché. Le chercheur me dit qu'il était temps de partir et m'indiqua ce que je devais retenir de cet épisode. Nous repartîmes donc, et je ne remis plus jamais les pieds dans ce marché. En dépit du fort sentiment de honte d'avoir participé à ces interrogatoires abrupts, cette leçon de terrain a confirmé à mes yeux l'importance de la mise en confiance des interlocuteurs, du temps passé à parler de sujets pas nécessairement liés à la migration, et la difficulté d'enregistrer et, à plus forte raison, de filmer les entretiens.

Enfin, le dernier lieu reste le hasard des rues et les rencontres fortuites. En effet, beaucoup de balayeurs de rue et de casseurs de glace des trottoirs moscovites sont centrasiatiques. Mais les interroger pose deux problèmes non négligeables.

Tout d'abord, il s'agit de reconnaître des migrants à leur faciès, ce qui, au-delà de poser un problème déontologique majeur, en pose un pratique : comment reconnaître avec certitude un Ouzbek à sa physionomie ? En réalité, ce ne sont bien sûr pas des critères naturels ou biologiques qui sont en jeu, puisqu'une ethnie n'existe pas en soi mais comme construction. Il s'agit bel et bien de critères culturels, et des attitudes corporelles qui trahissent la culture d'origine: la manière de s'accroupir pour se reposer est presque emblématique du travailleur ouzbek ${ }^{32}$, celle de porter la main au cœur en parlant, les façons de se saluer, de croiser les mains derrière le dos et tant d'autres, mêlées aux bribes de conversations entendues ${ }^{33}$.

Le second problème, moins évident, réside dans la difficulté à entrer en conversation avec un parfait inconnu, surtout quand il s'agit de l'interroger sur sa vie. De plus, parler dans la rue en russe pose un évident problème de confidentialité, puisque cette langue est a priori comprise par tous les passants. Or, de nombreux Ouzbeks éprouvent une grande gêne à exercer de tels métiers et n'ont guère envie d'en parler à une étrangère.

28 J'ai également pu visiter certains chantiers de construction et m'entretenir avec des ouvriers. De plus, l'appartement où j'étais hébergée, près de Krasnye vorota ${ }^{34}$, était en rénovation pendant mon travail de terrain et des travailleurs ouzbeks y participaient. J'ai pu m'entretenir quotidiennement avec eux, alors que la plupart du temps, je n'osais pas interrompre dans leur travail ceux que j'apercevais sur les chantiers, de peur que leur chef ne les réprimande.

29 Enfin, je dois évoquer trois derniers espaces sociaux, où je pensais pouvoir établir des contacts, et qui se sont révélés peu concluants. Tout d'abord, le champ religieux ne m’a été d'aucun secours car les migrants rencontrés ne se réunissaient pas pour prier et les 
mosquées moscovites ${ }^{35}$ n'étaient pas visitées par beaucoup de Centrasiatiques. Ensuite, celui des associations s'est aussi avéré maigre. La maison des nationalités m’a renvoyée vers Oleg Belkov, président de l'Uzbekskoe sodružestvo Moskvy (Association ouzbèke de Moscou). C'est la seule association en lien avec l'Ouzbékistan que j'ai trouvée, mais elle n'accueillait presque que des Russes, au grand regret des organisateurs. Les migrants rencontrés m'ont expliqué par la suite ne pas avoir le temps de se réunir, ni l'envie de le faire, par peur d'être plus visibles et donc en danger, devenant la cible potentielle des nationalistes russes. L'ambassade d'Ouzbékistan à Moscou ${ }^{36}$ s'est avérée être un lieu de recherche difficile: j'ai été repérée, suspectée puis arrêtée par les policiers de garde à l'entrée pour être passée deux fois dans la même journée devant eux. J'ai pu interroger de manière très succincte un employé du service consulaire, mais il n'était pas particulièrement loquace et s'est montré fort peu disposé à m'informer sur les migrations centrasiatiques en Russie.

Ces différents lieux m'ont donc permis de rencontrer des migrants, mais l'atmosphère de peur et de suspicion restait particulièrement présente et pesante lors de ce terrain.

\section{Travailler à Moscou}

31 Obtenir un salaire plus élevé que dans leur pays est la principale raison pour laquelle les Ouzbeks migrent à Moscou. Certains migrants partent en sachant où ils vont travailler, soit parce qu'ils sont passés par des agences spécialisées en Ouzbékistan qui proposent leurs services pour travailler en Russie, soit parce qu'ils ont mobilisé des relations dans le pays d'accueil pour trouver un emploi. Mais que font les migrants, les plus nombreux, qui arrivent seuls dans le pays d'accueil et n'ont pas de contact particulier avec de potentiels employeurs?

Certains journaux éditent des petites annonces qui proposent, entre autres, des emplois, par exemple, le magazine Rabota i Zarplata [r.: Travail et Salaire] en Russie. Mais en réalité, les offres d'emploi circulent le plus souvent par bouche à oreille ou par annonces directement affichées sur le lieu de travail. Ravšan raconte : «J'ai rejoint mon frère aîné à Moscou et, au début, j'ai fait des petits boulots, comme ça, au jour le jour. Puis un ami m'a dit que la Čajhana $n^{\circ} 1^{37}$ recherchait un serveur, et c'est comme ça que je suis venu ici. »

33 Le bouche à oreille semble transmettre des informations liées aux possibilités d'embauche et, pour certains emplois, une forme de chaîne de travail s'instaure où se relaient des migrants provenant tous d'Ouzbékistan. Tous mes interlocuteurs ouzbeks à Moscou étaient payés en argent liquide. Certains étaient rémunérés au jour le jour, d'autres chaque semaine, d'autres encore tous les quinze jours ou tous les mois.

D'après les médias, certains employeurs exigent de l'argent de la part des migrants avant de les faire travailler. On peut effectivement s'interroger sur le sens de cette transaction entre l'employeur et le salarié, dans le sens inverse de ce qui est attendu. Il est fort probable qu'il s'agisse d'extorquer abusivement de l'argent, ce dont sont parfois victimes les migrants ouzbeks à Moscou. Finalement, en règle générale, trouver un emploi ne semble pas poser de problèmes majeurs, aucun migrant ne m'ayant dit avoir été inactif pendant une période longue, mais la nature du travail est bien souvent délicate. 
En effet, les emplois occupés par les migrants, au moins à leur arrivée dans le pays d'accueil, sont le plus souvent ceux dont les autochtones ne veulent pas ou bien ceux dont ils ne suffisent pas à satisfaire l'offre. Il s'agit généralement d'emplois jugés dégradants et éreintants par ceux qui les refusent. Ils sont fréquemment sous-qualifiés, physiquement éprouvants, et parfois dangereux pour la santé. Beaucoup sont du travail clandestin, c'est-à-dire à la fois non déclaré et réalisé par des travailleurs en situation irrégulière sur le territoire d'accueil. Il existe plusieurs pôles principaux d'emploi pour les migrants ouzbeks rencontrés: les usines, les chantiers de construction, la manutention, les services à la personne, la restauration.

Un article traitant des produits en vente dans le marché de Čerkizogo estime que « tous les pulls, jeans et bottes qui s'entassent sur les comptoirs sont neufs, fabriqués par des couturières chinoises ou turques et importés en Russie essentiellement en fraude. Selon de nombreux témoignages, une grande partie de ces vêtements sont cousus dans des ateliers clandestins de la banlieue de Moscou, où des femmes tadjiks et ouzbeks travaillent jour et nuit pour un bol de kacha. Mais ces données sont difficiles à confirmer ${ }^{38} »$.

7 Les salaires divergent énormément suivant les emplois, mais la majorité des interlocuteurs que j'ai rencontrés travaillaient pour environ 200 à 300 dollars par mois, qu'ils soient balayeurs de rue, ouvriers dans des chantiers de construction, ou serveurs dans les restaurants. À Moscou, en mars 2008, le salaire minimum était de 6100 roubles [soit 166 euros]. Les migrants gagnaient donc à peu près l'équivalent du salaire minimum. De la même façon, j'ai été étonnée d'apprendre que Rustam, qui proposait de fausses cartes d'immigration et disait travailler pour son propre compte, ne gagnait lui aussi qu'entre 200 et 300 dollars selon les mois. S'agit-il de la somme qui lui restait après avoir acheté la bienveillance des policiers? travaillent dans le commerce, mais je n'en ai rencontré aucun: ce sont mes interlocuteurs qui ont évoqué cette voie possible pour gagner davantage. L'exemple marginal était celui de Behruz, gardien à l'accueil de l'ambassade d'Ouzbékistan de Moscou, qui m'a dit être rémunéré à hauteur de 1500 dollars par mois et être logé par l'ambassade.

39 Dans l'ensemble, les migrants démarrent leur vie à l'étranger en exerçant un travail peu attractif, mal rémunéré, physiquement éprouvant et symboliquement dégradant. Mais certains d'entre eux, après quelque temps et une meilleure connaissance du pays d'accueil, s'orientent vers d'autres types d'emplois.

\section{Que représente le travail pour les migrants?}

Au départ, les candidats à la migration pensent ne partir que pour amasser de l'argent et revenir en Ouzbékistan, une fois des économies substantielles réalisées. Ils n'y vont pas pour s'installer, ne prennent pas en considération la différence culturelle probablement induite par l'exil, ne mettent pas en avant l'appréhension de la découverte d'un nouveau monde. Ils n'entendent pas être altérés par cette expérience, estimant uniquement accomplir leur besogne, aussi rude soit-elle, et repartir. Mais comme le dit l'expression populaire française, « on n'arrive jamais pour les raisons qu'on avait de partir ». Une fois sur place, il paraît évident que la migration ne peut 
être vécue seulement comme une parenthèse, une suspension où le migrant n'entrerait pas réellement dans le pays d'accueil, restant dans sa bulle de travail.

Comme le notent avec justesse R. Bennegadi et $\mathrm{F}$. Bourdillon, « Quand on se réfère à la plupart des textes concernant le travailleur migrant, on retrouve d'une manière générale la mise en équation : vie du migrant = vie du travail, qui, extrapolée, justifie la vie du migrant seulement par son travail et exclut toute la dimension d'un projet, d'un scénario migratoire où le travail ne serait qu'un élément. À cette confusion entre l'homo-faber et l'homo-migrantus répondent d'autres analyses plus fines qui essaient de repérer la motivation de la migration et le rôle de la recherche du travail, la représentation sociale du travail dans la culture d'origine du migrant, la finalité de ce travail et sa valeur affective et symbolique ${ }^{39}$ ».

En réalité, bien que s'intégrer ne soit pas leur objectif, les migrants semblent marqués par cette période et par leur rapport au travail, qui n'est pas le même qu'en Ouzbékistan. Il est dès lors intéressant de s'interroger sur la vision que les migrants de travail ont d'eux-mêmes et de leur labeur. Dans de nombreux récits, le travail apparaît comme une activité éreintante, les journées étant longues, mais cette fatigue physique est présentée comme salvatrice, en ce qu'elle «empêche de penser ». C'est ce dont se souvient Âršigul, qui travaillait à Moscou :

J'ai passé un an à Moscou [en 2002-2003]. J'y étais allée pour rejoindre mon mari, car ce n'est pas bon de laisser les hommes trop longtemps tout seuls, tu comprends? [rires] Il travaille comme boulanger, avec ses frères, mais il est géologue de formation. Je suis revenue en Ouzbékistan car il voulait que je m'occupe des enfants. Mais moi, je voudrais retourner là-bas. C'est bizarre, quand j'y étais, je voulais être en Ouzbékistan et depuis que je suis à Samarkand, je voudrais être à Moscou. On vivait dans un quartier pauvre, dans une obŝežitie [r. : habitation collective], à côté de la boulangerie et à dix minutes de mon travail. Maintenant, il vit avec ses frères dans un appartement [que j'ai pu voir à Moscou], c'est mieux je pense.

Je travaillais d'abord comme femme de ménage chez des nouveaux riches, des banquiers, et puis j'ai été vendeuse dans un magasin. Ici, j'ai ma famille bien sûr, mais je m'ennuie. Je pourrais travailler à l'hôpital, puisque je suis infirmière, mais ça ne rapporte rien et je suis là pour m'occuper des enfants. Là-bas, je travaillais tellement que ma tête ne marchait plus! [rires] Je n'avais pas le temps de penser à mes enfants, j'étais comme saoule de fatigue. Je travaillais de neuf heures du matin à neuf heures du soir, sans interruption. J'avais beaucoup de mal à me lever le matin, et les premières heures de travail étaient les plus difficiles. Après, ça devient automatique. C'est ça, le travail là-bas : on s'assomme, mais au moins, on s'occupe.

Le discours de Âršigul est une réminiscence du passé migratoire et non un récit en migration. Elle compare donc sa situation actuelle, de mère au foyer habitant chez ses beaux-parents à Samarkand, à son année de travail à Moscou. Le travail y représentait une occupation aliénante, occupant toute la journée et ne laissant de répit que le soir, où le sommeil réparateur remplit le reste du temps. Travailler devient dès lors une échappatoire aux pensées nostalgiques.

L'emploi, à travers sa rémunération, est la priorité des migrants économiques, mais constitue parallèlement une bouée de sauvetage quotidienne permettant de lutter contre les fantômes de l'absence. Le travail constitue l'essentiel des journées des migrants rencontrés et laisse peu de place aux loisirs. La quasi-totalité de mes interlocuteurs travaillait au moins dix heures par jour. Les personnes qu'ils côtoyaient étaient principalement leurs collègues de travail. Le travail crée des réseaux sociaux 
particuliers: c'est un lieu d'interaction. Alors même que de nombreux migrants estiment n'être là que pour travailler, ils se voient néanmoins impliqués dans des enjeux sociaux et sont concernés par de nouveaux codes relationnels. Le travail est-il une façon de s'intégrer dans la société d'accueil ou au contraire, favorise-t-il l'enfermement dans le microcosme des travailleurs clandestins et étrangers? Il est d'usage de souligner, comme le fait J. Fayolle, que « l'accès à l'emploi joue [...] un rôle déterminant dans la qualité de l'intégration au sein du pays d'accueil ${ }^{40}$ ».

Pourtant, les interlocuteurs rencontrés à Moscou insistent sur le peu d'espace de liberté qu'ils s'autorisent, en raison de l'importance donnée au travail et de leur peur des mauvaises rencontres, avec la police ou avec des personnes xénophobes. Le travail n'est donc pas nécessairement pour eux un facteur d'intégration dans la société d'accueil, mais peut être un lieu de rencontres privilégié. Parallèlement, le travail en lui-même ne façonne pas l'identité, mais implique une identification particulière par la société d'accueil. Ainsi, se présenter comme ouvrier ou comme balayeur implique des représentations particulières de soi-même et évoque un système de référents préconçus chez l'interlocuteur. Mais c'est surtout le fait de n'être pas en règle avec l'administration locale qui marque le plus le rapport du migrant au pays d'accueil.

\section{Travailler illégalement : les risques encourus et les stratégies utilisées}

46 Nombre de mes interlocuteurs étaient clandestins, c'est-à-dire qu'ils n'étaient pas autorisés légalement à résider sur le territoire du pays d'accueil. À Moscou, beaucoup n'étaient pas enregistrés légalement, comme il se doit pour tous les non-Russes présents depuis plus de trois jours sur le territoire de la Fédération.

Parallèlement, la grande majorité n'avait pas d'autorisation de travail. F. Weber souligne dans un ouvrage intitulé Le travail au noir : une fraude parfois vitale? combien les termes de travail « au noir » ou « clandestin » véhiculent de connotations négatives. Si l'on reprend la distinction qu'elle opère dans ce livre, on peut dire que les migrants ouzbeks sont pour beaucoup des «travailleurs dissimulés ${ }^{41}$ ». En Russie, il faut obtenir un permis de travail mais le plus souvent les employeurs n'effectuent pas les démarches nécessaires pour les travailleurs illégaux venus d'Asie centrale. Les risques encourus en cas de violation de la législation diffèrent selon les cas.

Les contrôles policiers sont redoutés par l'ensemble des migrants ouzbeks qui dénoncent unanimement la traque dont ils sont victimes. Le " délit de faciès » est en effet monnaie courante parmi la milice russe, qui arrête de manière quasi systématique toute personne au physique non slave, et en particulier ceux désignés par le péjoratif čêrnye [r. : litt., noirs], c'est-à-dire particulièrement les personnes originaires d'Afrique noire, du Caucase et d'Asie centrale. Officiellement, les étrangers interpellés qui s'avèrent en situation irrégulière risquent une amende et l'expulsion. Dans les faits, il est rare que ces sanctions soient infligées, les agents russes préférant généralement les pots-de-vin à une procédure officielle, pénible et qui ne leur rapporte rien. De plus, la nouvelle loi votée en 2006 facilite l'obtention d'un permis de travail ${ }^{42}$.

49 Mais surtout, le rapport de l'État aux clandestins est ambigu: si l'illégalité est officiellement dénoncée, elle joue aussi un rôle économique important. Le caractère irremplaçable des migrants en Russie est ainsi souligné par O. Neterebski : «Dans un 
certain nombre de métropole, si tous les migrants décidaient de faire grève, certains secteurs économiques se trouveraient paralysés. Les trolleybus ne sortiraient plus des dépôts, les ordures ne seraient plus enlevées, tous les chantiers s'arrêteraient ${ }^{43}$ \%.

Enfin, il semble que la conduite du gouvernement face aux migrants soit parfois dictée par les aléas des relations internationales avec les pays pourvoyeurs de main-d'œuvre. En 2007, les Géorgiens installés, légalement ou non, en Russie, ont subi des représailles en raison d'un conflit politique entre Tbilissi et Moscou. Un rapport du Human Rights Watch a signalé que "la Russie prend les Géorgiens pour cibles d'expulsion. Le gouvernement a pris ce groupe ethnique pour cible en guise de réponse au conflit politique $^{44}$ ». Les migrants servent alors à la fois de boucs émissaires mais aussi d'un moyen de pression dont dispose le pays d'accueil pour soumettre le pays d'origine.

Ainsi, malgré la nécessité économique de recourir à une main-d'œuvre étrangère et le caractère indispensable des migrants non régularisés, les migrants ouzbeks rencontrés redoutent les contrôles policiers et leurs incidences sur leur migration. Face à ces risques (être arrêté et sanctionné par une amende ou une expulsion), les migrants mettent en place des stratégies de contournement des obstacles législatifs, soit en tentant d'échapper aux contrôles, soit en essayant de limiter leurs conséquences négatives. Une des stratégies couramment usitée consiste à éviter les rencontres avec les policiers et autres contrôleurs. C'est ce qu'expliquent certains vendeurs dans les marchés de Moscou, comme Zulhumor, vendeuse tadjike au marché de Teplyj stan ${ }^{45}$ :

Les gens qui contrôlent sur les marchés, on les voit venir... On connaît à peu près leurs horaires. Certains [commerçants, d'origine centrasiatique pour la plupart], pour ne pas avoir de problème, préfèrent fermer leur stand quand ils les savent dans le marché. Mais la plupart du temps, il faut payer quelque chose...

Depuis que la loi sur la préférence nationale a été votée à la Douma le 8 décembre 2006, réservant aux Russes $50 \%$ des places de marché, de nombreux commerçants centrasiatiques essayent de trouver une vendeuse russe, pour la désigner comme responsable de l'étal en cas de contrôle. Dans ce cas, les migrants connaissent la loi et s'en servent habilement pour être dans la légalité tout en maintenant leurs activités commerciales; ils la détournent ainsi de son but initial, qui était de réduire le nombre de commerçants non Russes sur les marchés.

53 À Moscou, les migrants ne semblent pas craindre particulièrement les contrôles au travail, qu'ils considèrent comme un des moyens permettant aux policiers de leur soutirer régulièrement de l'argent. En Russie, la corruption permet donc le plus souvent d'éviter le renvoi en Ouzbékistan et, même en cas d'expulsion, le retour est possible et peu onéreux. Plus grave est le risque d'accidents de travail, car, en cas d'emploi clandestin, ceux-ci ne sont couverts par aucune assurance et laissent les migrants blessés sans ressources ni recours. À ces accidents survenus directement sur le lieu de travail s'ajoutent les problèmes de santé liés à la pénibilité des emplois occupés, des troubles qui peuvent se déclarer bien plus tard, parfois quand le migrant est de retour dans son pays d'origine.

Par ailleurs, des migrants rentrent de Russie en ayant subi des amputations, des fractures osseuses et autres atteintes physiques plus ou moins graves, sans que les familles apprennent nécessairement ce qui est à l'origine de ces traumatismes corporels. 


\section{Des carrières migratoires?}

55 Il peut paraitre étonnant de parler de "carrières migratoires ». En effet, le terme de « carrière professionnelle » est plus souvent employé pour des parcours de vie. Mais il correspond bien à l'évolution professionnelle de certains de mes interlocuteurs en migration, qui relatent d'ailleurs leur expérience en termes d'ascension. Ainsi, parallèlement à la carrière migratoire ascendante dans le pays d'accueil se développe une carrière internationale. Celle-ci serait évaluable à l'aune d'une échelle communément admise, qui classerait les destinations de la moins enviable à la plus attrayante : Kazakhstan, Russie, Corée, Europe, Etats-Unis.

Pour la majorité de mes interlocuteurs, cependant, l'évolution de leur carrière migratoire, en termes d'emploi et de pays d'accueil, n'est que la poursuite du but initial: gagner de l'argent pour pouvoir ensuite réintégrer le pays d'origine. La question qui se pose ne porte que sur la durée de la période migratoire. De nombreux migrants n'imaginent pas, au départ, rester si longtemps loin de leur famille. Mais la connaissance du pays d'accueil et le tissage de relations en migration permettent une évolution qui empêche le migrant de repartir en arrière, conscient qu'il est du parcours accompli. Un migrant installé depuis longtemps peut monnayer ses connaissances.

En outre, l'argent économisé en Russie sert parfois au migrant à repartir, vers une destination qu'il juge plus lucrative. En effet, il semble délicat de parler d'un véritable choix de la destination. En réalité, il s'agit plutôt de savoir combien d'argent est réuni pour le projet migratoire. Plus la somme dont peut disposer le candidat à la migration est élevée, plus il pourra s'élever sur l'échelle des destinations : Moscou pour les moins argentés, Séoul pour ceux qui ont réussi à mobiliser un important réseau social, New York pour les plus riches. À ces degrés d'investissement, correspond une hiérarchisation des salaires. Il est courant d'entendre dire que, parmi ces trois destinations, les salaires les moins élevés (200-400 dollars mensuels) sont perçus à Moscou, les moyens sont touchés à Séoul (entre 800 et 1000 dollars mensuels) et les plus élevés à New York (entre 1000 et 2000 dollars mensuels) ${ }^{46}$. Il ne s'agit donc pas à proprement parler de migration économique, puisque les plus pauvres ne peuvent partir pour les destinations jugées lucratives. Il existe donc des carrières migratoires qui visent à partir d'Ouzbékistan pour s'enrichir le plus possible, afin de revenir au pays d'origine avec un statut plus avantageux qu'avant. Il s'agit donc bien d'un paradoxe : " partir à l'étranger pour mieux vivre en Ouzbékistan ». 


\section{ANNEXES}

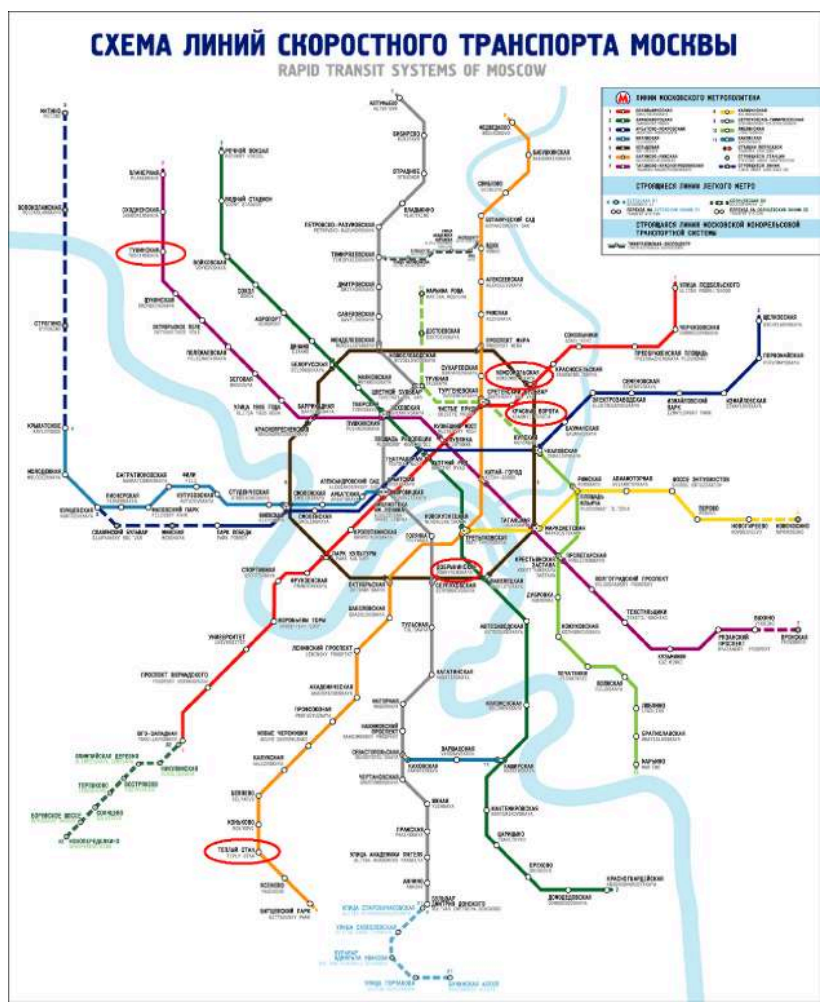

Plan du métro de Moscou, où sont entourées les stations citées dans l'article

\section{NOTES}

1. Cet article est issu de recherches effectuées dans le cadre d'un doctorat de science politique. La thèse, dirigée par D. Colas, s'intitule Enjeux politiques et identitaires de la migration internationale des Ouzbeks vers trois pôles urbains (Moscou, Séoul, New York) et a été soutenue à l'Institut d'études politiques de Paris en septembre 2009.

2. Il convient ici de distinguer le terme «ouzbek» (qui renvoie à la nationalité) et « ouzbékistanais » (qui renvoie à la citoyenneté).

3. Un soulèvement populaire a vu le jour à Andijan, dans la vallée de Ferghana, le 13 mai 2005. Les causes en sont encore imprécises, en raison de la censure exercée par le pouvoir, qui a interdit l'accès de la zone aux journalistes et bloqué les connexions Internet aux sites étrangers pour que les Ouzbékistanais n'en soient pas tenus informés. Il semble que l'origine de cette révolte soit à chercher dans l'incarcération d'opposants politiques, dont les proches réclamaient la libération. A cette revendication concernant une sortie de prison s'est ajoutée l'expression d'un désarroi général des habitants, qui se sont joints aux manifestants. Islam Karimov a alors ordonné la répression de cette insurrection par les forces armées, qui ont tiré sur la foule. Le bilan humain est particulièrement lourd, bien que difficilement chiffrable en raison de la chape de plomb qui pèse sur la région depuis ces événements Le gouvernement ouzbek a reconnu 169 morts, mais les opposants au régime de Karimov et les ONG présentes en Ouzbékistan estiment que ce chiffre pourrait en réalité s'élever jusqu'à un millier. Cette hécatombe a placé quelque temps l'Ouzbékistan sous les lumières internationales, et le gouvernement ouzbékistanais a été vivement critiqué par l'OSCE et l'OTAN, mais soutenu par la Fédération de Russie. 
4. Voir http://www.ladocumentationfrancaise.fr/monde/chronologies/russie-cei-2005.shtml.

5. La Csтo (Collective Security Treaty Organization [en russe, ОDKB : Organizaciâ Dogovora o Kollektivnoj Bezopasnosti])a pour membres la Russie, la Biélorussie, l'Arménie, le Kazakhstan, le Kirghizstan, le Tadjikistan et l'Ouzbékistan.

Voir Vladimir Socor, «Uzbekistan accedes to collective security treaty organization », Eurasia Daily Monitor,disponible sur : http://www.jamestown.org/edm/article.php?article_id=2371223.

6. L'EurASEC (Eurasian Economic Community [en russe, EvrAzÉS: Evrazijskoe ékonomičeskoe soobŝestvo])a pour membres la Russie, la Biélorussie, le Kazakhstan, le Kirghizstan, le Tadjikistan et l'Ouzbékistan.

7. Voir https://www.cia.gov/library/publications/the-world-factbook/geos/uz.html.

8. En $2006,23,9 \%$ des exportations d'Ouzbékistan se faisaient en direction de la Russie et $27,8 \%$ des importations vers l'Ouzbékistan provenaient de Russie, selon le World Factbook (https:// www.cia.gov/library/publications/the-world-factbook/geos/uz.html).

9. http://www.septimanie-export.com/fr/fiches-pays/ouzbekistan/accueil.

10. https://www.cia.gov/library/publications/the-world-factbook/geos/uz.html.

11. http://www.tlfq.ulaval.ca/axl/Asie/ouzbekistan.htm.

12. http://www.bibliomonde.net/pages/fiche-livre.php3?id_ouvrage=3915\&texte_aff=infocomp.

13. http://www.tlfq.ulaval.ca/axl/Asie/ouzbekistan.htm.

14. Scott Radnitz, "Weighing the political and economic motivations for migration in PostSoviet space : The case of Uzbekistan », Europe-Asie Studies, vol. 58, n $5: 659$.

15. Johann Uhres, "Ouzbékistan, une langue difficile à imposer ", Le Courrier des pays de l'Est, $\mathrm{n}^{\circ} 1018,2001: 18-26$.

16. http://www.levoyageur.net/television.php?pays=Russie.

17. http://www.ntv.ru/.

18. L'abréviation « 0 . » désignera désormais les termes ouzbeks, l'abréviation « $r$. " les termes russes. Pour la translittération des termes russes, je me suis référée à la norme ISO 9 : 1995. Par ailleurs, il convient de noter que le genre n'existe pas dans les langues turques; l'utilisation d'articles masculins ou féminins en français est donc arbitraire.

19. J'ai choisi de préserver l'anonymat des personnes rencontrées en leur attribuant de façon aléatoire dans ces pages des prénoms ouzbeks. En effet, parler à une étrangère de sujets sensibles est possible en Ouzbékistan (mes séjours me l'ont prouvé), mais risque néanmoins d'entraîner des conséquences fâcheuses pour ceux qui se confient ainsi. De la même façon, dans les pays d'accueil, parler des phénomènes migratoires n'est pas sans danger pour les migrants en situation illégale. Nombre de mes interlocuteurs m'ont ainsi dit qu'ils craignaient d'être repérés par les services de migration chargés des contrôles des étrangers. Plusieurs m'ont confié leur crainte que je sois un espion du gouvernement ouzbek ou du pays d'accueil. Enfin, certains redoutaient que je cite leur nom dans un média français et que cette mention ne les empêche à tout jamais de gagner l'Europe.

20. Le site d'Uzbekistan Airways indique, comme distance à vol d'oiseau, 2547 kilomètres entre Tachkent et Moscou : http://www.visit-uzbekistan.com/uzbekistan/flights/aircrafts.php.

21. Les plus fréquents sont le train et l'avion, mais le bus et la voiture sont aussi utilisés par certains. Des bus partent des principales villes d'Ouzbékistan, pour se rendre à Moscou. Le train est un moyen de transport davantage utilisé, semble-t-il, par les migrants qui partent en Russie que par ceux qui en reviennent. Ces derniers craignent les pillards qui les détroussent fréquemment, les laissant au mieux sans argent, au pire sans vie. Les trains passent par le Kazakhstan, et c'est cette traversée qui apparaît périlleuse aux yeux des Ouzbeks.

22. Le plus onéreux est l'avion. Il fallait compter environ 500 dollars pour un vol TachkentMoscou sur Aéroflot à l'époque du terrain (prix moyen donné à titre indicatif, car les tarifs évoluent en fonction des dates de vol et de la compagnie aérienne). 
23. Ces recherches étaient menées, dans le cadre du doctorat, dans une visée comparatiste, raison pour laquelle je me suis aussi rendue à Séoul en juillet-août 2006 et à New York en févriermars 2007, mais ces deux volets ne sont pas l'objet du présent article.

24. Métro 1 ou 5, arrêt Komsomol'skaâ. Un plan du métro de Moscou est disponible en annexe. J'ai choisi ce repère spatial dans la ville qui est celui généralement utilisé par les migrants pour se situer. Les logements des migrants rencontrés se situaient pour beaucoup dans la périphérie de Moscou, à l'extrémité des lignes de métro. Les rares qui habitaient à l'intérieur du kol'co [r. : litt., cercle ; à Moscou, évoque une frontière officieuse, matérialisée par la ligne de métro 5 nommée Kol'cevaâ et des boulevards périphériques, séparant le cœur de la capitale russe de ses faubourgs] vivaient sur leur lieu de travail.

25. La registraciâ $[r$. : enregistrement administratif] est obligatoire pour tout étranger qui pénètre dans le territoire de la Fédération de Russie. Il dispose de trois jours pour se faire enregistrer par les autorités compétentes. Cela constitue un obstacle administratif important pour de nombreux ressortissants centrasiatiques, qui ne parviennent pas toujours à le surmonter. Beaucoup vivent et travaillent illégalement à Moscou.

26. Restaurants tels que Čajhana $n^{\circ} 1$ [r. : Maison de thé $\left.n^{\circ} 1\right]$ dans le parc Gorkii (CPK i OIM Gor'kogo), Hodža Nasreddin v Hive [r. : Nasredin Hodja à Khiva] (10, ul. Pokrovka), Uzbekistan [r. : Ouzbékistan] (29, ul. Neglinnaâ).

27. Principalement le Tušinskij bazar [métro 7, arrêt Tušinskaâ] et celui de Teplyj stan [métro 6, arrêt Teplyj stan]

28. Cependant, une loi sur la préférence nationale a été votée à la Douma le 8 décembre 2006 et vise à favoriser les citoyens russes de nationalité russe. Désormais, $50 \%$ des places de marché sont réservées aux paysans et producteurs russes, et $20 \%$ des places des marchés vestimentaires aux Russes « de souche ». Cette loi semble difficilement applicable.

29. Plat à base de riz, de carottes, d'oignons et de viande, typique de l'Asie centrale.

30. Métro 6, arrêt Teplyj stan.

31. Cette question renvoie à la registraciâ [ $r$. : enregistrement administratif].

32. Voir par exemple la caricature "The eXile Field Guide to Moscow: Uzbek Gasterbeiter, Artwork by Roman Papsuev», disponible sur: http://www.exile.ru/articles/detail.php? ARTICLE_ID=7443\&IBLOCK_ID=35.

33. Je ne parle pas ouzbek mais peux reconnaître certains termes employés couramment.

34. L'arrêt de métro Krasnye vorota se situe sur la ligne 1.

35. «Aujourd'hui Moscou compte près de 2 millions d'adeptes de l'islam, sur une population totale de 8533 millions ( $1^{\mathrm{er}}$ janvier 2003)». Disponible sur: http://oumma.com/article.php3? id_article $=1063$.

Il y a actuellement cinq mosquées dans la capitale russe. Voir http://www.imam.ru/mecheti/ moskva.html.

36. Ambassade d'Ouzbékistan à Moscou (Posol'stvo Uzbekistana) : Pogorel'skij pereulok, 12 [métro 5, arrêt Dobrinskâa].

37. Čajhana $n^{\circ} 1\left[r\right.$. : Maison de thé $\left.n^{\circ} 1\right]$ : restaurant qui se situe dans le parc Gorkii (CPK i OIM Gor'kogo),

38. http://www.ruvr.ru/main.php?lng=fre\&q=1355\&cid=78\&p=23.10.2007.

39. Rachid Bennegadi, François Bourdillon, «La santé des travailleurs migrants en France: aspects médico-sociaux et anthropologiques ", Revue européenne des migrations internationales, vol. 6, n 3, 1990 : 129.

40. Jacky Fayolle, "Migrations anciennes et nouvelles. Les politiques et les acteurs à l'épreuve », Chronique internationale de l'IRES, $\mathrm{n}^{\circ} 84$, dossier « Mouvements et politiques migratoires, les enjeux sociaux », septembre 2003: 8

41. Florence Weber, Le travail au noir : une fraude parfois vitale? Paris, Éditions Rue d'Ulm, 2008. 
42. "Since 2006, the Russian authorities have become aware of the importance of regulating migratory flows, and accordingly they passed legislation which aims to select immigrants. The new law on migrants was voted on July 18, 2006, and entered into effect on January 15, 2007. It reduces the requirements for registration and obtaining a work permit for those migrants who cross, or crossed, the border legally. It does not, however, normalize those already present on Russian territory without formal status. More than 700,000 foreigners received work permits in 2006 - a number which remains small compared to the millions of illegal immigrants living in Russia. » (Marlène Laruelle, «Central Asian labor migrants in Russia : The "diasporization" of the Central Asian States?», China and Eurasia Forum Quarterly, vol. 5, n 3, 2007 : 113)

43. Oleg Neterebski, «Les problèmes migratoires en Russie », Éducation ouvrière, n 129 (4), 1992 : 150.

44. http://www.hrw.org/french/docs/2007/10/01/russia17006.htm.

45. Métro 6, arrêt Teplyj stan.

46. Il s'agit de la moyenne des salaires déclarés par les migrants rencontrés lors des différents terrains. Pour la différence de salaire d'un manœuvre dans les trois pays d'accueil, voir : http://www.statistiques-mondiales.com/manoeuvre_instituteur.htm.

INDEX

Thèmes : migration, mobilité

Mots-clés : enquête, réseau, carrière migratoire

Index géographique : Moscou, Russie

\section{AUTEUR}

SOPHIE MASSOT

Docteur en science politique, Ater de science politique à l'Université de la Réunion, sophiealdric@yahoo.fr 